\title{
Atividade antifúngica de óleos essenciais frente a amostras clínicas de Candida albicans isoladas de pacientes HIV positivos
}

\begin{abstract}
ALMEIDA, L.F.D. ${ }^{1}$; CAVALCANTI, Y.W. ${ }^{2}$; CASTRO R.D. ${ }^{3}$; LIMA E.O. ${ }^{4}$
${ }^{1}$ Departamento de Odontologia Restauradora, Faculdade de Odontologia de Araraquara, Universidade Estadual Paulista, Rua Humaitá, 1680, CEP:14801-903 , Araraquara-Brasil ${ }^{2}$ Departamento de Prótese e Periodontia, Faculdade de Odontologia de Piracicaba, Universidade de Campinas, Avenida Limeira, 901, CEP: 13414-903, Piracicaba-Brasil ${ }^{3}$ Departamento de Clínica e Odontologia Social, Universidade Federal da Paraíba, Cidade Universitária CEP: 58051-900, João Pessoa-Brasil ${ }^{4}$ Departamento de Ciências Farmacêuticas, Universidade Federal da Paraíba, Cidade Universitária, CEP: 58051-900, João Pessoa-Brasil *leopoldinalmeida@hotmail.com
\end{abstract}

RESUMO: Objetivou-se avaliar a atividade antifúngica dos óleos essenciais de Ocimum basilicum L. (manjericão), Cymbopogon martinii L. (palmarosa), Thymus vulgaris L. (tomilho) e Cinnamomum cassia Blume (canela da china) sobre cepas de Candida albicans isoladas de pacientes HIV positivos e cepa padrão (ATCC 76845). Quinze amostras clínicas de $C$. albicans (C1-C15) foram repicadas em ágar Sabouraud Dextrose, para confecção de suspensões em solução salina estéril

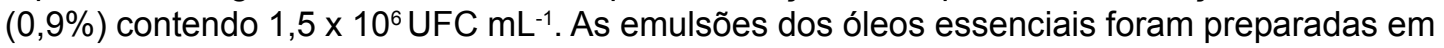
água destilada estéril e tween 80 , com concentrações variando entre $1024 \mu \mathrm{g} \mathrm{mL}^{-1} \mathrm{e}_{4} \mu \mathrm{g} \mathrm{mL}^{-1}$. A ação antifúngica foi determinada por meio da Concentração Inibitória Mínima (CIM) utilizando-se a técnica da microdiluição. Foram utilizados como controles positivos a nistatina e o miconazol $\left(50 \mu \mathrm{g} \mathrm{mL}^{-1}\right)$. Os testes foram realizados em triplicata, sendo a CIM, a menor concentração capaz de inibir o crescimento das leveduras, observada por método visual de acordo com a turvação do meio de cultura. Para C. albicans (ATCC 76845), a CIM do óleo essencial de C. cassia foi $64 \mu \mathrm{g} \mathrm{mL}^{-1}$, enquanto para óleo de $C$. martinii foi $1024 \mu \mathrm{g} \mathrm{mL}^{-1}$. Para as cepas clínicas, verificou-se que a CIM de C. cassia para $80 \%$ das cepas foi $64 \mu \mathrm{g} \mathrm{mL}^{-1}$, sendo a variação dos valores da CIM entre $128 \mu \mathrm{g} \mathrm{mL}^{-1}$ e $64 \mu \mathrm{g} \mathrm{mL}^{-1}$. Observou-se que para 66,6\% das amostras clínicas, a CIM de C. martinii foi $612 \mu \mathrm{g} \mathrm{mL}^{-1}$. Constatou-se que a nistatina não apresentou atividade frente às cepas clínicas (C1-C15), enquanto a atividade antifúngica do miconazol foi verificada em $100 \%$ das amostras. Não se constatou atividade antimicrobiana dos óleos essenciais de $O$. basilicum e T. vulgaris, nas concentrações avaliadas. Concluiu-se que os óleos essenciais de $C$. cassia e C. martinii, em diferentes concentrações, apresentam atividade antifúngica sobre cepas de C. albicans isoladas de pacientes HIV positivos e cepa padrão (ATCC 76845). Entretanto não foi observada inibição antimicrobiana para os óleos de $O$. basilicum e $T$. vulgaris.

Palavras- chave: Candida albicans, produtos naturais, tomilho, manjericão, canela

ABSTRACT: Antifungal activity of essential oils against clinical samples of Candida albicans isolated from HIV-positive patients. The aim of this study was to evaluate the antifungal activity of essential oils from Ocimum basilicum L. (basil), Cymbopogon martinii L. (palmarosa), Thymus vulgaris L. (thyme) and Cinnamomum cassia Blume (Chinese cinnamon) against Candida albicans strains isolated from HIV-positive patients and the standard strain (ATCC 76845). Fifteen clinical samples of $C$. albicans (C1-C15) were subcultured in Sabouraud Dextrose agar to prepare suspensions in sterile saline solution $(0.9 \%)$ containing $1.5 \times 10^{6} \mathrm{CFU} \mathrm{mL}{ }^{-1}$. The emulsions of essential oils were prepared in sterile distilled water and Tween 80 , with concentrations ranging between $1024 \mu \mathrm{g} \mathrm{mL}^{-1}$ and $4 \mu \mathrm{g} \mathrm{mL}^{-1}$. The antifungal action was determined by means of the Minimum Inhibitory Concentration (MIC), using the microdilution technique. Nystatin and miconazole $\left(50 \mu \mathrm{g} \mathrm{mL}^{-1}\right)$ were used as positive controls. The tests were performed in triplicate and the MIC was the lowest concentration capable of inhibiting the growth of yeasts, which was observed by the visual method, according to the turbidity of the culture medium. For C. albicans (ATCC 76845), the MIC of $C$. cassia essential oil was $64 \mu \mathrm{g} \mathrm{mL}^{-1}$, while the MIC for $C$. martini was $1024 \mu \mathrm{g} \mathrm{mL}^{-1}$. Considering the clinical strains, the MIC of C. cassia was $64 \mu \mathrm{g} \mathrm{mL}^{-1}$ for $80 \%$ of the strains, and

Recebido para publicação em 11/05/2011

Aceito para publicação em 25/04/2012

Rev. Bras. PI. Med., Botucatu, v.14, n.4, p.649-655, 2012. 
the variation in MIC values was between $128 \mu \mathrm{g} \mathrm{mL}^{-1}$ and $64 \mu \mathrm{g} \mathrm{mL}^{-1}$. For $66.6 \%$ of the clinical samples, the MIC of C. matinii was $612 \mu \mathrm{g} \mathrm{mL}^{-1}$. Nystatin did not present activity against the clinical strains (C1-C15), while the antifungal activity of miconazole was noticed for $100 \%$ of the samples. The antimicobrial activity of essential oils from $O$. basilicum and $T$. vulgaris was not identified at the evaluated concentrations. It was concluded that the essential oils from C. cassia and $C$. martinii, at different concentrations, presented antifungal activity against $C$. albicans strains isolated from HIV-positive patients and the standard strain (ATCC 76845). However, antifungal activity was not observed for the essential oils from $O$. basilicum and $T$. vulgaris.

Key words: Candida albicans, natural products, thyme, basil, cinnamon

\section{INTRODUÇÃO}

A microbiota oral normal apresenta, regularmente, espécies de Candida, sem haver comprometimento da saúde do indivíduo (Kuriyama et al., 2005). Entretanto, por ser uma levedura oportunista, pode causar candidose oral, devido ao rompimento das relações comensais existentes (Crocco et al., 2004). Fatores como baixo fluxo salivar (Torres et al., 2007) e a utilização inadequada de próteses dentárias podem acentuar a infecção fúngica em pacientes portadores de doenças como a Síndrome da Imudeficiência Adquirida (AIDS) (Noborikawa et al., 2009). Assim, entre indivíduos portadores do vírus da imunodeficiência (HIV), a candidose oral é uma infecção comum e a levedura Candida albicans consiste na espécie mais prevalente (Gabler et al., 2008; Pozzatti et al., 2008).

Dentre os fármacos mais utilizados para o tratamento da candidose oral, destacam-se os derivados azólicos (fluconazol, miconazol e itraconazol) e os poliênicos (anfotericina B e nistatina), atuando de forma tópica ou sistêmica (Kuriyama et al., 2005). Entretanto, observa-se certa resistência das espécies de Candida, principalmente aquelas que infectam pacientes HIV positivos, a alguns destes medicamentos (Rex et al., 2000). Assim, frente à resistência das espécies de Candida aos antifúngicos sintéticos observa-se o uso de produtos naturais, na tentativa de obter-se melhor desempenho sobre tais microrganismos (Lima et al., 2006 ; Pozzati et al., 2008).

Os óleos essenciais como os de Cymbopogon martinii (palmarosa), Ocimum basilicum (manjericão) e Thymus vulgaris (tomilho) apresentam atividade frente a espécies bacterianas e fúngicas, incluindo a C. albicans (Duarte et al., 2005; Almeida et al., 2010; Cavalcanti et al., 2011). A ação antibacteriana e antifúngica de espécies como Cynnamomum zeylanicum (canela), Eucaliptus citriodora (eucalipto) e Eugenia uniflora (pitanga) também foram relatadas por Araújo et al. (2004).

O tratamento da candidose oral em pacientes infectados pelo HIV é instituído empiricamente e por longo tempo, na maioria dos casos. Este fato contribui para o aparecimento de espécies resistentes à terapia medicamentosa comum (Wingeter et al., 2007). Tal evento tem motivado estudos sobre a susceptibilidade in vitro de fungos, isolados principalmente de pacientes imunocomprometidos, a novos produtos devido ao aparecimento de espécies resistentes (Bachmann, 2002; Almeida et al., 2010).

Diante disto, objetivou-se determinar a Concentração Inibitória Mínima dos óleos essenciais de Ocimum basilicum L. (manjericão), Cymbopogon martinii L. (palmarosa), Thymus vulgaris L. (tomilho) e Cinnamomum cassia Blume (canela da china) frente a cepas de $C$. albicans, isoladas de pacientes HIV positivos e cepa padrão (ATCC 76845).

\section{MATERIALE MÉTODO}

\section{Seleção das leveduras}

O presente estudo foi submetido e aprovado pelo Comitê de Ética em Pesquisa do Hospital Universitário Lauro Wanderley de acordo com a resolução 196/96.

Foram incluídas no estudo 15 amostras clínicas de $C$. albicans (CA 01- CA 15) isoladas de pacientes portadores de HIV e, devidamente identificadas, escolhidas aleatoriamente da micoteca do Laboratório de Micologia da Universidade Federal da Paraíba, João Pessoa, Paraíba. Utilizou-se cepa padrão de C. albicans - CAP (ATCC 76845).

As amostras das leveduras foram repicadas em ágar Sabouraud Dextrose (DIFICO ${ }^{\circledR}$, Detroit, Michigan, EUA) e incubadas em estufa a $35^{\circ} \mathrm{C}$ por 48 horas. As suspensões fúngicas foram preparadas utilizando-se solução salina estéril a $0,9 \%$, sendo comparadas ao tudo 0,5 da escala de MacFarland $\left(1,5 \times 10^{6} \mathrm{UFC} \mathrm{mL} \mathrm{mL}^{-1}\right)$.

\section{Soluções dos óleos essenciais}

Os óleos essenciais de Ocimum basilicum (manjericão), Cymbopogon martinii (palmarosa), Thymus vulgaris (tomilho) e Cinnamomum cassia (canela da china) foram obtidos, mediante compra, através da Empresa Viessence ${ }^{\circledR}$ (Florianópolis, Santa 
Catarina, Brasil). As propriedades físico-químicas foram descritas pelo fornecedor por meio de laudo técnico (Tabela 1).

Para cada óleo essencial, foi preparada uma solução padrão, levando-se em consideração valor médio de densidade de $0,9 \mathrm{~g} \mathrm{~mL}^{-1}$. A solução final, foi constituída de $0,011 \mathrm{~mL}$ do óleo essencial, 0,04 mL TWEEN 80 (Farmafórmula Farmácia de Manipulação, João Pessoa-PB, Brasil) e q.s.p. $5 \mathrm{~mL}$ de água destilada estéril. Assim, foi estabelecida uma concentração inicial padrão de $2048 \mu \mathrm{g} \mathrm{mL}^{-1}$, para todos os produtos.

\section{Concentração Inibitória Mínima (CIM)}

A atividade antifúngica, determinada pela Concentração Inibitória Mínima, foi avaliada por meio da técnica da microdiluição em caldo, utilizando-se microplacas com 96 orifícios, com fundo em forma de "U”, com tampa (ALAMAR ${ }^{\circledR}$, Diadema, São Paulo, Brasil).

Inicialmente, foram distribuídos $100 \mu \mathrm{L}$ de caldo Sabouraud Dextrose (DIFICO ${ }^{\circledR}$, Detroit, Michigan, EUA) duplamente concentrado nos orifícios das placas de microdiluição. Em seguida, $100 \mu \mathrm{L}$ das soluções padronizadas dos óleos essenciais foram adicionadas na linha $A$, sendo o conteúdo dos orifícios homogeneizados com o meio e transferidos para o orifício da linha seguinte (B), repetindo-se este procedimento até a linha $\mathrm{H}$, de modo a obter uma concentração decrescente, que variou entre $1024 \mu \mathrm{g}$ $\mathrm{mL}^{-1}$ e $4 \mu \mathrm{g} \mathrm{mL}^{-1}$. Os $100 \mu \mathrm{L}$ finais foram desprezados. Nos orifícios de cada coluna foram dispensadas alíquotas de $10 \mu \mathrm{L}$ do inóculo correspondente a cada cepa a ser avaliada (CLSI, 2002). Paralelamente, foram realizados controle da viabilidade das cepas de leveduras. Realizou-se controle de sensibilidade das cepas frente à ação antifúngica do miconazol e nistatina, em uma concentração fixa de $50 \mu \mathrm{g} \mathrm{mL}^{-1}$.

Os ensaios foram realizados em triplicata e incubados a $35^{\circ} \mathrm{C}$ durante 48 horas. A leitura para determinação da CIM dos óleos sobre as cepas de leveduras foi realizada a partir do método visual. Assim, considerou-se a formação ou não de aglomerados de células ("botão") no fundo da cavidade da placa. A CIM foi determinada como a menor concentração das amostras em teste capaz de produzir inibição visível sobre o crescimento das cepas de leveduras utilizadas nos ensaios microbiológicos (CLSI, 2002). Diante disto, os dados foram analisados descritivamente.

\section{RESULTADO}

Os resultados referentes à CIM dos óleos essenciais estão dispostos na Tabela 2. Observouse que todas as cepas avaliadas foram sensíveis aos óleos de C. cassia (canela) e C. martinii (palmarosa). Em relação aos controles com os antifúngicos, observou-se que $100 \%$ das amostras foram sensíveis ao miconazol, entretanto, observou-se crescimento fúngico de todas as cepas para a nistatina.

\section{DISCUSSÃO}

A atividade antifúngica dos óleos essenciais de C. martinii (palmarosa), O. basilicum (manjericão) e $T$. vulgaris (tomilho) foi avaliada frente a cepas de C. albicans (ATCC 10231) por Sartorato et al. (2004) e Duarte et al. (2005). Dos estudos, observou-se que a CIM dos óleos de C. martinii e $O$. basilicum foi superior a $2000 \mu \mathrm{g} \mathrm{mL}^{-1}$, considerando-os de baixa atividade; já para o T. vulgaris foi de $2000 \mu \mathrm{g} \mathrm{mL}^{-1}$, sendo apresentado como produto de moderada atividade antifúngica. Entretanto observando os valores da CIM do óleo essencial de T. vulgaris (tomilho) frente à mesma cepa, Rahimifard et al. (2008) encontraram CIM de $0,3 \mu \mathrm{g} \mathrm{mL}^{-1}$.

Comparando-se os resultados obtidos neste estudo observa-se que os valores médios de CIM (612$256 \mu \mathrm{g} \mathrm{mL}^{-1}$ ) alcançados pelo óleo essencial da $C$. martinii frente às cepas, foi consideravelmente menor que o valor encontrado por Sartorato et al. (2004) e Duarte et al. (2005). Os óleos essenciais de T. vulgaris e $O$. basilicum não produziram inibição sobre 0 crescimento das leveduras mesmo diante da concentração inicial empregada $\left(1024 \mu \mathrm{g} \mathrm{mL}^{-1}\right)$, não apresentando similaridade com os resultados apresentados por Sartorato et al. (2004), Duarte et al. (2005) e Rahimifard et al. (2008).

As diferenças entre os valores de CIM do

TABELA 1. Especificações técnicas dos óleos essenciais utilizados no estudo, segundo laudo técnico expedido por fornecedor.

\begin{tabular}{llll}
\hline Óleos essenciais & $\begin{array}{l}\text { Densidade } \\
\left(\mathbf{g ~ m L} \mathbf{~}^{-1}, \mathbf{2 0}\right.\end{array}$ & Origem & Fitoconstituintes \\
\hline Ocimum basilicum (manjericão) & 0,898 & Brasil & Linalol1,8-cineol \\
Cymbopogon martinii (palmarosa) & 0,878 & Brasil & GeraniolAcetato de geranila \\
Thymus vulgaris (tomilho) & 0,908 & França & Timolp-cimeno \\
Cinnamomum cassia (canela da china) & 0,912 & China & Aldeído cinâmico \\
\hline
\end{tabular}

Rev. Bras. Pl. Med., Botucatu, v.14, n.4, p.649-655, 2012. 
TABELA 2. Concentração Inibitória Mínima $(\mu \mathrm{g} \mathrm{mL}-1)$ dos óleos essenciais frente cepas de $C$. albicans clínicas e padrão.

\begin{tabular}{|c|c|c|c|c|c|c|}
\hline \multirow{2}{*}{$\begin{array}{c}\text { Cepas de } C \text {. } \\
\text { albicans }\end{array}$} & \multicolumn{4}{|c|}{ Óleos essenciais } & \multirow{2}{*}{ Miconazol } & \multirow{2}{*}{ Nistatina } \\
\hline & O. basilicum & C. martinii & T. vulgaris & C. cassia & & \\
\hline CA- 01 & + & 256 & + & 64 & 50 & + \\
\hline CA- 02 & + & 256 & + & 64 & 50 & + \\
\hline CA- 03 & + & 256 & + & 64 & 50 & + \\
\hline CA- 04 & + & 256 & + & 64 & 50 & + \\
\hline CA- 05 & + & 612 & + & 64 & 50 & + \\
\hline CA- 06 & + & 256 & + & 64 & 50 & + \\
\hline CA- 07 & + & 612 & + & 64 & 50 & + \\
\hline CA- 08 & + & 612 & + & 64 & 50 & + \\
\hline CA- 09 & + & 612 & + & 64 & 50 & + \\
\hline CA- 10 & + & 612 & + & 64 & 50 & + \\
\hline CA- 11 & + & 612 & + & 64 & 50 & + \\
\hline CA- 12 & + & 612 & + & 128 & 50 & + \\
\hline CA- 13 & + & 612 & + & 64 & 50 & + \\
\hline CA- 14 & + & 612 & + & 64 & 50 & + \\
\hline CA- 15 & + & 612 & + & 64 & 50 & + \\
\hline ATCC 76845 & + & 1024 & + & 64 & 50 & + \\
\hline
\end{tabular}

${ }^{*}+$ Crescimento microbiano. Ausência de atividade antifúngica a partir de $1024 \mu \mathrm{g} \mathrm{mL}^{-1}$

óleo essencial de T. vulgaris, mesmo nos achados que utilizaram a mesma cepa de $C$. albicans, podem ser atribuídas às condições climáticas e disponibilidade de água no solo, que afetam o metabolismo secundário da espécie vegetal, podendo alterar a composição dos óleos essenciais, de mesma espécie, nas diferentes estações do ano (Freire et al., 2006). Atrelado a este fato, pode-se observar que os estágios fenológicos e clico de reprodução podem interferir na concentração dos fitoconstituintes (Carvalho et al., 2008).

Mesmo observando-se a ausência de atividade antifúngica do óleo essencial de $O$. basilicum no presente estudo, deve-se ressaltar as características antioxidante, moduladora da síntese de colesterol (Bravo et al., 2008) e antimicrobianas frente à Staphylococcus aureus, Escherichia coli e Aspergillus niger (Hussain et al., 2008).

O extrato bruto etanólico do T. vulgaris foi avaliado frente a cepas de C. albicans, C. glabrata e C. parapsilosis nas concentrações de 50, 100 e 200 $\mathrm{mg} \mathrm{mL}^{-1}$ por Silva \& Rangel (2010), por meio de difusão em ágar. Os autores constataram que a concentração de $50 \mathrm{mg} \mathrm{mL}^{-1}$ foi a mais efetiva sobre C. albicans, considerando-se a metodologia empregada. Nas condições do presente estudo, não foi observada atividade antifúngica para o óleo de $T$. vulgaris.

Assim, a comparação entre os resultados deve se feita com ressalvas, pois os requisitos metodológicos dos estudos foram distintos. Além disto, os veículos utilizados e as formas de extração do princípio ativo dos produtos, extrato bruto etanólico e o óleo essencial, poderia interferir na ação antifúngica, havendo diferença na concentração do princípio ativo responsável pela atividade anti-Candida.

Outro componente encontrado no óleo essencial de $T$. vulgaris, o carvacrol, tem sido citado como indutor de células apoptóticas no carcinoma hepatocelular (Yin et al., 2012). Além disto, alguns óleos essenciais e extratos, pertencentes a espécies da família Lamiaceae são relatados como antioxidantes. Assim, intensifica-se a pesquisa a cerca de tais substâncias potencialmente empregadas na indústria alimentícia (Mauritti \& Bragagnolo, 2007).

A atividade antifúngica do carvacrol e timol, isolados de $T$. viciosoi, foi avaliada frente a cepas de C. albicans. O estudo de Vale-Silva et al. (2010) demonstrou que a concentração inibitória mínima e fungicida mínima variaram entre 0,04 a $0,64 \mu \mathrm{L} \mathrm{mL}^{-1}$, demonstrando potencial antifúngico. Neste estudo, o óleo essencial de $T$. vulgaris não apresentou atividade inibitória, dentro das concentrações avaliadas. Tais diferenças podem ser justificadas pela análise antifúngica de espécies vegetais diferentes e ausência da análise sobre os principais fitoconstituintes.

Gioradani et al. (2004) avaliaram a atividade 
antifúngica de alguns óleos essenciais, dentre estes do óleo essencial de $T$. vulgaris, com percentual de $63,2 \%$ de timol, isolado e em associação a anfotericina $b$, frente à $C$. albicans (ATCC 90029). Os autores verificaram a CIM do referido óleo essencial na concentração $0,016 \%$, para $80 \%$ das amostras fúngicas, sendo analisado que a associação do óleo essencial ao antifúngico poderá ser utilizada em futuros tratamentos de micoses. Assim, observa-se a necessidade de ensaios posteriores que objetivem 0 isolamento dos fitoconstituintes dos óleos essenciais e possíveis associações aos antifúngicos sintéticos, bem como o emprego de outras cepas fúngicas, que apresentem fenótipos distintos.

A atividade antimicrobiana do óleo essencial da C. martinii foi constatada frente a microrganismos como S. aureus, E. coli, $P$. aeruginosa, $S$. thyphimurium e $C$. perfringens, observando valores de CIM entre 0,4 e 0,8 $\mathrm{mg} \mathrm{mL}^{-1}$ (Scherer et al., 2009). O mesmo óleo essencial teve atividade comprovada frente ao $S$. cerevisiae na concentração de 0,1\% (Prashar et al., 2003). Assim, constata-se a eficácia antimicrobiana de C. martinii frente a espécies bacterianas e fúngicas, como observa-se no presente estudo, com CIM variando entre 1024 e $256 \mu \mathrm{g} \mathrm{mL}^{-1}$. Esta atividade está relacionada à presença de geraniol e acetato de geralniol, compostos com elevada atividade antimicrobiana (Prashar et al., 2003; Duarte et al., 2005).

A atividade antifúngica dos óleos de $O$. basilicum e $T$. vulgaris não foram observadas nas concentrações testadas, entretanto a ação antimicrobiana destes produtos, em concentrações diferentes, foi constatada em alguns estudos (Sartorato et al., 2004; Duarte et al., 2005; Rahimifard et al., 2008; Lixandru et al., 2010), sendo esta atividade atribuída ao eugenol, presente em plantas do gênero Ocimum (Pereira \& Maia, 2007) e à presença de timol, em maior quantidade no óleo essencial de $T$. vulgaris, mas também encontrado no óleo de O. basilicum (Sartorato et al., 2004).

A atividade antifúngica dos óleos essenciais de O. basilicum e T. vulgaris foi avaliada por Lixandru et al. (2010) pelo método da Concentração Bactericida Mínima, frente a C. albicans (ATCC 10231) utilizandose o meio líquido Mueller Hinton, com concentração inicial de $8 \%$ do óleo, em volume. Observou-se atividade antifúngica dos óleos em concentrações de $0,25 \%$ e $0,06 \%$, para $O$. basilicum e $T$. vulgaris, respectivamente (Lixandru et al., 2010). A comparação entre os estudos deve ser realizada com distinção, pois observa a utilização de meios de culturas diferentes nos estudos, o que pode propiciar diferenças nos crescimentos das cepas, as quais também apresentavam composição fenotípica distintas.

Dentre os produtos avaliados, o óleo essencial de $C$. cassia apresentou os menores valores de CIM, variando entre 128 e $64 \mu \mathrm{g} \mathrm{mL}-1$, sendo o mais eficaz entre as amostras. Assim, estes achados corroboram com os resultados encontrados por Giordani et al. (2006), que avaliaram a atividade do óleo essencial de $C$. cassia frente a $C$. albicans (ATCC 90029), de forma isolada e associado à Anfotericina B. O produto foi caracterizado como um eficiente antifúngico, sendo verificada a CIM igual a $0,169 \mu \mathrm{L} \mathrm{mL}-1$, para $80 \%$ das amostras,

O óleo essencial de C. cassia foi avaliado frente ao crescimento de hifas e esporos de Aspergillus niger. Observou-se que o óleo essencial, comparado a outras 74 amostras de óleos teve o melhor desempenho, relacionando-se os valores dos halos de inibição das amostras, sendo inferior apenas ao óleo essencial de $C$. zeylanicum (Pawar \& Thaker, 2006).

No referido estudo a composição química do óleo foi analisada, destacando-se a presença do cinanomaldeído $(66.36 \%)$ e eugenol $(7.74 \%)$, conhecidos pela atividade antifúngica. (Pawar \& Thaker, 2006). É válido ressaltar que o estudo citado utilizou metodologia e cepa fúngica distintas daquelas utilizadas por este, entretanto a ação do óleo essencial da canela foi constatada.

A atividade antimicrobiana de fitoconstituintes extraídos do óleo essencial de $C$. cassia, foi descrita por Ooi et al. (2006). Foram avaliados o ácido cinâmico, o cinanomaldeído e óleo essencial sobre bactérias Gram positivas, Gram negativas, fungos dermatófitos e leveduras, dentre estas a $C$. albicans. Os autores observaram que a CIM do cinanomaldeído foi de $125 \mathrm{mg} \mathrm{mL}^{-1}$ e para o óleo essencial bruto o valor observado foi de $138 \mathrm{mg} \mathrm{mL}^{-1}$ e finalmente para o ácido cinâmico não foi encontrado valor de CIM. Desta forma, ao comparar os resultados aqui apresentados observa-se aproximação entre os valores de CIM (128-64 $\left.\mu \mathrm{g} \mathrm{mL}^{-1}\right)$, reforçando o potencial antifúngico do óleo essencial estudado. É válido ressaltar que as diferenças metodológicas encontradas nos estudos podem interferir nos valores de CIM, pois naquele realizado por Ooi et al. (2006) utilizou-se a técnica da difusão em ágar, entretanto no presente estudo realizou-se a técnica da microdiluição.

A atividade antimicrobiana do óleo essencial da C. cassia foi testada para Escherichia coli, Enterococcus sp., Klebsiella sp. e Proteus mirabilis, sendo a CIM do óleo, frente a E. coli e P. mirabilis, $0,1 \mathrm{mg} \mathrm{mL}^{-1}$ e $0,001 \mathrm{mg} \mathrm{mL}^{-1}$, respectivamente (Carvalho et al., 2010). Assim, mesmo comparando espécie fúngicas e bacterianas, os resultados se assemelham aos observados por Carvalho et al. (2010), pois a CIM frente às bactérias foi de $0,1 \mathrm{e}$ $0,001 \mathrm{mg} \mathrm{mL}^{-1} \mathrm{e}$ a CIM sobre as cepas de $C$. albicans variou entre 128 e $64 \mu \mathrm{g} \mathrm{mL}^{-1}$. Portanto, verifica-se

Rev. Bras. PI. Med., Botucatu, v.14, n.4, p.649-655, 2012. 
que esse produto natural, conforme observado em outros estudos, possui ação antimicrobiana efetiva, em baixas concentrações.

É importante ressaltar que poucos estudos a cerca da atividade antifúngica do óleo essencial de C. cassia foram encontrados na literatura, caracterizando o presente ensaio como pioneiro a abordar a atividade antifúngica do óleo essencial frente a C. albicans padrão e potencialmente virulentas.

Os óleos essenciais são conhecidos pelo caráter hidrofóbico, o que permite interação com estruturas lipídicas, aumentando a permeabilidade celular, provocando danos irreversíveis à célula (Nascimento et al., 2007). Tal constatação poderia indicar possível mecanismo de ação dos óleos essenciais de C. cassia (canela) e C. martinii (palmarosa), os quais apresentaram resultados satisfatórios frente às amostras de $C$. albicans.

Entretanto, observa-se que as realizações de testes microbiológicos e farmacológicos complementares tornam-se relevantes diante da necessidade de se estabelecer o real mecanismo de ação dos produtos frente às cepas de $C$. albicans e outras espécies de microrganismos potencialmente patogênicos.

\section{CONCLUSÃO}

Concluiu-se que os óleos essenciais de $C$. cassia e C. martinii, em diferentes concentrações, apresentam atividade antifúngica sobre cepas de $C$. albicans isoladas de pacientes HIV positivos e cepa padrão (ATCC 76845). Entretanto não foi observada atividade antimicrobiana para os óleos de $O$. basilicum e T. vulgaris.

\section{REFERÊNCIA}

ALMEIDA, L.F.D. et al. Screening da atividade antifúngica de óleos essenciais sobre Candida albicans. Revista Brasileira de Ciências da Saúde, v.14, n.4, p.51-6, 2010. AMRANI, S. et al. Hypolipidaemic activity of aqueous Ocimum basilicum extract in acute hyperlipidaemia induced by triton WR-1339 in rats and its antioxidant property. Phytotherapy Research, v.20, n.12, p.1040-5, 2007.

ARAÚJO, J.C.L.V. et al. Ação antimicrobiana de óleos essenciais sobre microrganismos potencialmente causadores de infecções oportunistas. Revista de Patologia Tropical, v.33, n.1, p.55-64, 2004.

BACHMANN, S.P. In vitro activity of caspofungin (MK0991) against Candida albicans clinical isolates displaying different mechanisms of azole resistance. Journal Clinical Microbiology, v.40, n.6, p.2228-30, 2002. BRAVO, E. et al. Ocimum basilicum ethanolic extract decreases cholesterol synthesis and lipid accumulation in human macrophages. Fitoterapia, v.79, n.7-8, p.51523, 2008.
CARVALHO, C.A. et al. Estudo espectrométrico de diferentes estágios fenológicos da Brassica oleracea var. capitata. Revista Brasileira de Farmacognosia, v.18, n.2, p.249-57, 2008.

CARVALHO, T.M. et al. Verification of the antibacterial activity in vitro of essential oils of Cinnamomum zeylanicum and Rosmarinus officinalis in bacteria which cause infections of the urinary tract. Revista Brasileira de Análises Clínicas, v.42, n.3, p.213-5, 2010.

CAVALCANTI, Y.W. et al. Efeito inibitório de óleos essenciais sobre microrganismos do canal radicular. Revista de Odontologia da UNESP, v.40, n.5, p.226-33, 2011.

CLSI. National Committee for Clinical Laboratory Standards. Norma M27-A2. Método de referência para testes de diluição em caldo para determinação da sensibilidade de leveduras à terapia antifúngica. 2.ed. Pennsylvania: NCCLS; 2002. 51p.

CROCCO, E.I. et al. Identificação de espécies de Candida e susceptibilidade antifúngica in vitro: estudo de 100 pacientes com candidíases superficiais. Anais Brasileiros de Dermatologia, v.79, n.6, p.689-97, 2004. DUARTE, M.C.T. et al. Anti-Candida activity of essential oils and extracts from native and exotic medicinal plants used in Brazil. Journal of Etnopharmacology, v.97, n.2, p.305-11, 2005.

FREIRE, C.M.M. et al. Effects of seasonal variation on the central nervous system activity of Ocimum gratissimum L. essential oil. Journal of Ethnopharmacology, v.105, n.1/2, p.161-6, 2006.

GABLER, I.G. et al. Incidence and anatomic localization of oral Candidiasis in patients with aids hospitalized in a public hospital in Belo Horizonte, MG, Brazil. Journal of Applied Oral Science, v.16, n.4, p.247-50, 2008.

GIORDANI, R. et al. Antifungal effect of various essential oils against Candida albicans. Potentiation of antifungal action of amphotericin b by essential oil from Thymus vulgaris. Phytotherapy Research, v.18, n.12, p.990-5, 2004. GIORDANI, R. et al. Potentiation of antifungal activity of amphotericin b by essential oil from Cinnamomum cassia. Phytotherapy Research, v.20, n.1, p.58-61, 2006. HUSSAIN, A.I. et al. Chemical composition, antioxidant and antimicrobial activities of basil (Ocimum basilicum) essential oils depends on seasonal variations. Food Chemistry, v.108, n.1, p.986-95, 2008.

KURIYAMA, T. et al. In vitro susceptibility of oral Candida to seven antifungal agents. Oral Microbiology and Immunology, v.20, n.6, p.349-53, 2005.

LIMA, I.O. et al. Atividade antifúngica de óleos essenciais sobre espécies de Candida. Revista Brasileira de Farmacognosia, v.16, n.2, p.197-201, 2006.

LIXANDRU, B.E. Antimicrobial activity of plant essential oils against bacterial and fungal species involved in food poisoning an/or food decay. Romanian Archives of Microbiology and Immunology, v.69, n.4, p.224-30, 2010. MARIUTTI, L.R.B.; BRAGAGNOLO, N. Revisão: antioxidantes naturais da família Lamiaceae. aplicação em produtos alimentícios. Brazilian Journal Food Technology, v.10, n.2, p.96-103, 2007.

NASCIMENTO, P.F.F. et al. Atividade antimicrobiana dos óleos essenciais: uma abordagem multifatorial dos métodos. Revista Brasileira de Farmacognosia, v.17, n.1, p.108-13, 2007. 
NOBORIKAWA, E. et al. Biotypes of Candida albicans isolates from the oral mucosa of HIV seropositive and control subjects. Revista Odonto Ciência, v.24, n.3, p.258-63, 2009.

OOI, L.S.M. et al. Antimicrobial activities of cinnamon oil and cinnamaldehyde from the Chinese medicinal herb Cinnamomum cassia Blume. The American Journal of Chinese Medicine, v.34, n.3, p.511-22, 2006. PAWAR, V.C.; THAKER, V.S. In vitro efficacy of 75 essential oils against Aspergillus niger. Mycoses, v.49, n.4, p.316-23, 2006.

PEREIRA, C.A.M.; MAIA, J.F. Estudo da atividade antioxidante do extrato e do óleo essencial obtidos das folhas de alfavaca (Ocimum gratissimum L.). Ciência e Tecnologia de Alimentos, v.27, n.3, p.62432, 2007.

POZZATTI, P. et al. In vitro activity of essential oils extracted from plants used as aspices against fluconazole-resistent and fluconazole-susceptible Candida spp. Canadian Journal of Microbiology, v.54, n.6, p.950-6, 2008.

PRASHAR, A. et al. Antimicrobial action of palmarosa oil (Cymbopogon martinii) on Saccharomyces cerevisiae. Phytochemistry, v.63, n.5, p.569-75, 2003.

RAHIMIFARD, N. et al. Antifungal activity of the native essential oil of Thymus vulgaris on Candida albicans, Aspergillus niger and Aspergillus flavus from Iran. Journal of Pure and Applied Microbiology, v.2, n.2, p.343-6, 2008. REX, J.H. et al. Practice guidelines for the treatment of candidiasis. Journal of Infectious Diseases, v.30, n.4, p.662-78, 2000.

SARTORATTO, A. et al. Composition and antimicrobial activity of essential oils from aromatic plants used in Brazil. Brazilian Journal of Microbiology, v.35, n.4, p.27580, 2004.

SCHERER, R. et al. Composição e atividades antioxidante e antimicrobiana dos óleos essenciais de cravo-da-índia, citronela e palmarosa. Revista Brasileira de Plantas Medicinais, v.11, n.4, p.442-9, 2009.

SILVA, T.B.; RANGEL, E.T. Avaliação da atividade antimicrobiana do extrato etanólico do tomilho (Thymus vulgaris L.) in vitro. Revista Eletrônica de Farmácia, v.7, n.2, p.48-58, 2010.

TORRES, S.R. et al. prospective randomized trial to reduce oral Candida spp. colonization in patients with hyposalivation. Brazilian Oral Research, v.21, n.2, p.1827, 2007.

VALE-SILVA, L.A. et al. Antifungal activity of the essential oil of Thymus viciosoi against Candida, Cryptococcus, Aspergillus and dermatophyte species. Planta Medica, v.76, n.9, p.882-8, 2010.

WINGETER, M.A. et al. Identificação microbiológica e sensibilidade in vitro de Candida isoladas da cavidade oral de indivíduos HIV positivos. Revista da Sociedade Brasileira de Medicina Tropical, v.40, n.3, p.272-6, 2007. YIN, Q. et al. Anti-proliferative and pro-apoptotic effect of carvacrol on human hepatocellular carcinoma cell line HepG-2. Cytotechnology, v.64, n.1, p.43-51, 2012. 